\title{
A RELATIONSHIP BETWEEN PARABOLIC AND SPHERICAL FUNCTIONS
}

\section{HARRY HOCHSTADT}

One of the many formulas, which are usually classed under addition theorems, due to Gegenbauer, is that [1]

$J_{\nu-1 / 2}\left(r \sin \theta \sin \theta^{\prime}\right)\left(r \sin \theta \sin \theta^{\prime}\right)^{-\nu+1 / 2} \exp \left(i r \cos \theta \cos \theta^{\prime}\right)$

$$
=\frac{2^{2 \nu} \Gamma^{2}(\nu)}{(2 \pi)^{1 / 2}} \sum_{m=0}^{\infty} \frac{i^{m} m !(\nu+m)}{\Gamma(m+2 \nu)} \frac{J_{\nu+m}(r)}{r^{\nu}} C_{m}^{\nu}(\cos \theta) C_{m}^{\nu}\left(\cos \theta^{\prime}\right) .
$$

If a new parameter $t$ is introduced by the substitution

$$
\cos \theta^{\prime}=\frac{1-t}{1+t}, \quad \sin \theta^{\prime}=\frac{2 t^{1 / 2}}{1+t}
$$

and $\nu-1 / 2$ is replaced by $u$ one obtains immediately

$$
\begin{gathered}
\frac{J_{u}\left(\frac{2 p t^{1 / 2}}{1+t}\right) \exp \left(i z \frac{1-t}{1+t}\right)}{t^{u / 2}(1+t)}=\frac{2^{3 u} \Gamma^{2}(u+1 / 2)(\sin \theta)^{u}}{(2 \pi r)^{1 / 2}} \\
\times \sum_{m=0}^{\infty} \frac{i^{m} m !(2 m+2 u+1)}{\Gamma(m+u+1)} J_{u+m+1 / 2}(r) C_{m}^{u+1 / 2}(\cos \theta) \frac{C_{m}^{u+1 / 2}\left(\frac{1-t}{1+t}\right)}{(1+t)^{u+1}}
\end{gathered}
$$

where $p=r \sin \theta, z=r \cos \theta$.

The term on the left side of the equality is the generating function $G_{u}(P, t)$ of the parabolic functions [2]. This function expressed in parabolic coordinates is

$$
\begin{aligned}
G_{u}(P, t) & =\sum_{n=0}^{\infty} \Omega_{n}^{u}(P)(-t)^{n}, \\
\Omega_{n}^{u}(P) & =\frac{\Gamma(1+n+u)}{n !} m_{n+(1+u) / 2}^{u}(-2 i \xi) m_{n+(1+u) / 2}^{u}(2 i \eta), \\
\xi & =\frac{r+z}{2}, \quad \eta=\frac{r-z}{2}, \\
m_{n+(1+u) / 2}^{u}(z) & =\frac{z^{u / 2} e^{-z / 2}}{\Gamma(1+u)}{ }_{1}^{u} F_{1}(-n ; 1+u ; z) .
\end{aligned}
$$

Received by the editors July 27, 1956 and, in revised form, September 26, 1956. 
These are the functions which are obtained when the wave equation is solved by the method of separation of variables in parabolic coordinates.

The right side of equation (1) can be expanded into a power series in the parameter $t$ and comparison with equation (2) leads to the result

$$
\begin{aligned}
\Omega_{s}^{u}(P)=\sum_{n=0}^{\infty} \alpha(n ; u, s) \frac{J_{u+n+1 / 2}(r)}{(2 \pi r)^{1 / 2}} C_{n}^{u+1 / 2}(\cos \theta)(\sin \theta)^{u}, \\
s=0,1,2, \cdots, u \neq-1,-2,-3, \cdots
\end{aligned}
$$

where

$$
\begin{aligned}
\alpha(n ; u, s)=\frac{2^{3 u} \Gamma^{2}(u+1 / 2) i^{n}(2 n+2 u+1)}{\Gamma(1+2 u)} \\
\quad \times \sum_{r=0}^{s} \frac{(-)^{r}(-n)_{r}(n+2 u+1)_{r}(r+u+1)_{s-r}}{r !(s-r) !(u+1)_{r}} .
\end{aligned}
$$

It is desirable to invert the system of equations (3). The obvious approach of using the orthogonality of the Gegenbauer polynomials does not apply, because the resultant integrals are not convergent. Other standard techniques such as use of residue evaluations of contour integrals proved unfruitful and a purely algebraic technique was resorted to. Multiplication of equations (1) and (2) by $(1+t)^{u+1}$ and introduction of a new parameter $w$ defined by

$$
t=\frac{w}{1-w}
$$

leads, after expanding in a power series in $w$ and comparing coefficients, to

(4)

$$
\begin{aligned}
& \sum_{s=0}^{l}(-)^{s} \frac{(s+u+1)_{l-s}}{(l-s) !} \Omega_{s}^{u}(P) \\
& =\frac{2^{3 u} \Gamma^{2}(u+1 / 2)(\sin \theta)^{u}}{(2 \pi r)^{1 / 2} \Gamma(1+2 u) l !(u+1)_{l}} \\
& \times \sum_{n=l}^{\infty} i^{n}(2 n+2 u+1) J_{u+n+1 / 2}(r) C_{n}^{u+1 / 2}(\cos \theta)(-n)_{l}(n+2 u+1)_{l} \\
& \quad l=0,1,2, \cdots, u \neq-1,-2,-3, \cdots .
\end{aligned}
$$

The inverse to this system can now be stated 


$$
\begin{aligned}
& \frac{i^{s} J_{u+s+1 / 2}(r)}{(2 \pi r)^{1 / 2}} C_{s^{u+1 / 2}}^{u}(\cos \theta)(\sin \theta)^{u} \\
& =\sum_{n=s}^{\infty} \frac{(-)^{s} \Gamma(1+2 u)(u+1)_{n} 2^{-3 u} n !}{s !(n-s) !(s+2 u+1)_{n+1} \Gamma^{2}(u+1 / 2)} \\
& \quad \times \sum_{\sigma=0}^{n}(-)^{\sigma} \frac{(\sigma+u+1)_{n-\sigma}}{(n-\sigma) !} \Omega_{\sigma}^{u}(P) \\
& \quad s=0,1,2, \cdots, u \neq-1,-2,-3, \cdots .
\end{aligned}
$$

The proof of (5) follows from a substitution of (5) into (4) and use of

$$
\begin{aligned}
& \sum_{n=l}^{k} \frac{(2 n+2 u+1)(-n)_{l}(n+2 u+1)_{l}(-)^{n}}{n !(k-n) !(n+2 u+1)_{k+1}} \\
= & \frac{1}{(k-l) !(2 l+2 u+2)_{k-l}}\left[{ }_{2} F_{1}(-k+l, 2 l+2 u+2 ; k+l+2 u+2 ; 1)\right. \\
& \left.-\frac{k-l}{k+l+2 u+2}{ }_{2} F_{1}(-k+l+1,2 l+2 u+2 ; k+l+2 u+3 ; 1)\right] \\
= & \delta_{k l}
\end{aligned}
$$

An interchange of the summations in (5) is not permissible, because the double series is only conditionally convergent. A fuller discussion of this question can be found in reference (2), where this program was carried out for the special case where $u$ is an integer. There the results (3) and (5) are used to derive an addition theorem for the parabolic functions by referring the problem back to the corresponding addition theorem for spherical functions [3].

\section{REFERENCES}

1. G. N. Watson, Theory of Bessel functions, p. 37, Macmillan, 1944.

2. H. Hochstadt, Addition theorems for the functions of the paraboloid of revolution, Institute for the Mathematical Sciences, BR-18, May, 1956.

3. A. Erdélyi et al, Higher transcendental functions, vol. II, McGraw-Hill, 1953, p. 257.

W. L. Maxson CORP. 